\title{
Communication challenges with older adults patients during clinical learning: A qualitative study among students nurse in Indonesia
}

\author{
R Setiyani ${ }^{1 * 1}$, M Sumarwati ${ }^{1}$, A Iskandar ${ }^{1}$ and I Rismawati ${ }^{1}$ \\ ${ }^{1}$ Faculty of Health Sciences, Jenderal Soedirman University, Indonesia
}

\begin{abstract}
One of consequences of population aging is the increase of health care utilization by elderly population. Student nurse who will work as a professional nurse in the future are expected to be able to communicate effectively with elderly patients, in order to delivering high quality care. This research aimed at exploring communication challenges with older adult patients experienced by students nurse during clinical practice in Indonesia. In-depth interview conducted among 6 final year students nurse, who selected using purposive sampling method. Data were analyzed using Charmaz's constructivist grounded theory. There are four categories of participant's experience communicating with elderly patients were emerged from the data analysis: 1) "less functioning”; 2) 'kromo' language as a symbol of respect; 3) "bridging the gap" and 4) "intimate connection". The present findings revealed that communication challenges could be either from patient or nurse's students. Improving communication skills by learning the basics of elderly first language (Jawa "Kromo") are necessary to students nurse for an effective communication with elderly. Developing constructive view to elderly is also pivotal for successful intergenerational communication.
\end{abstract}

\section{Introduction}

The growing of elderly population worldwide has brought profound implications. One of the consequences of population aging is the increase in health care service utilization by older people, mostly due to aging related chronic diseases [1]. Longterm care service is also projected to be highly demanded [2]. Those situations lead to the increasing demand of health care workforce who will work closely to older people [1]. With no exception, most nurses will also be involved in caring for older people.

Communication is one of important aspects of patient care. Communication is involved throughout nursing process, starting from assessment to interventions, such as health promotion and education, prevention, treatment and rehabilitation [3].

*Corresponding author: rahmi.setiyani@unsoed.ac.id 
Nurses use communication as a means to establish helping relationship with patients and families [4].

Communication is also essential for greater patient outcome. There has been growing studies showing the benefits of effective communication between nurses and patients. A literature review concludes that patient-centered communication style has positive impacts to patient satisfaction to care services, patient health status and patient adherence to care plan [5].

Nurses are expected to develop good communication skills to all age groups considering its enormous benefits. However, communication to older people could be challenging. Communication becomes the major problem nurses have to face when caring for elderly with cognitive deficit such as dementia. There are lack of understanding of the meaning of message sent by the patient and of language agreement between nurse and patient [6]. Patient related factors are such as hearing deficit, being tired, mistrust, and memory problem and nurse related factors such as lack of intimacy, formal and insincere attitude has been identified to be barriers for effective communication between nurses and elderly patients [7].

Although many researches has been done to identify communication barrier to older adult, study that consider cultural related factor in communication is still rare. Indonesia is an example of multicultural society, which consists of many ethnic groups. Many of them share different value, belief and spoken languages.

Students nurse who will work as a professional nurse in the future are expected to be able to communicate effectively with elderly patients, in order to delivering high quality care. Thus, this research aims at exploring communication challenges with older adult patients during clinical practice of students nurse of a public university in Central Java, Indonesia.

\section{Methods}

\subsection{Study design}

In this qualitative study, students nurse were interviewed in depth about their experiences regarding communicating with older adult patients during clinical practice. Data were collected after students finished all clinical rounds to make sure that they have had experience caring for elderly patients. Then, data were analyzed using Charmaz' Constructivist Grounded Theory [8].

\subsection{Participants}

Participants are 6 final year nursing students of a public university in Central Java, Indonesia. This location was chosen since there are many students from various ethnic and cultural backgrounds attend this university, creating a culturally diverse society. Final year students were chosen as they have already has experience caring for patients from different age groups, including older people. They also have passed clinical practicums in various health care settings, including hospital, long term facilities, and communities. Participants were recruited using purposive sampling technique. 


\subsection{Ethical consideration}

Ethical approval for the research was gained from Ethics Committee of Faculty of Medicine of X University (Reg no. 0816060538). Participants were informed about aim and nature of the study, and the right to refuse and withdrawn from the participation at any time before signing the written consent. Confidentialy was secured by limiting access to the data only to reseachers. Initial was used to labeling the data to preserve anonymity.

\subsection{Data collection}

Interviews were conducted face to face in a mutually agreed upon setting after signing the informed consent. The interviews were conducted in Bahasa Indonesia and audiorecorded, each lasting 45 minutes to 60 minutes.

\subsection{Data analysis}

Constructivist grounded theory was used to guide data analysis. Analysis began through process of coding. Coding was conducted in 3 steps: initial coding, focused coding, and theoretical coding [8]. Information from 6 participants was considered adequate to generate categories. Interviewing more participants would not give more information nor generate new category.

\section{Results}

There are four categories that emerged from the data analysis: 1) "less comprehend and less able to hear”; 2) 'Kromo' language as a symbol of respect; 3) "bridging the gap" and 4) "intimate connection". Those categories depict perceived barriers, strategy to deal with challenges, and perceived nature of interaction:

\subsection{Category 1: Less functioning}

This category captures communication barriers as result of decline in physical functions, particularly in cognitive and sensory function. Elderly showed lower understanding to complex sentences and instruction compared to other age group as a result of cognitive decline. Hearing loss experienced by elderly was found to be another factor that makes communication become less effective.

"It is quite easy communicating with adult patients. When we talk about something, they usually understand what we mean. But, talking to elderly patients is different. Even when we have already used simple sentences, they still don't understand what we really mean (R.1)." (R.2).”

"Yes, they (elderly patients) sometimes couldn't really catch what we mean

"In my opinion, the biggest barrier (to interact with elderly patients) is in communication because their hearing function has already declined (R.2)."

"Hmm...mostly elderly patients suffer from hearing decline. That is the problem. Most often, they couldn't get what we really mean (R.6).", 


\subsection{Category 2: 'Kromo' language as a symbol of respect}

Javanesse is the spoken language speaks by most of elderly in this study. Javanesse is known to have different speech styles for different social context. In Java culture, people are expected to speech in "Kromo" style as symbol of respect when they communicate to people with "higher" social status, including to elderly. However, lack in "Kromo" language mastering by students nurse means lack in medium to deliver the symbol. Students in this study truly understand that the use of other languages which understood well by both parties such as Bahasa or Jawa Ngoko style would never be able to give the similar "sense of respect" as 'Kromo' language does.

"When we have to speak Kromo language, we find it is difficult to do. As opposite, when we speak Bahasa, they (the elderly patients) find difficulties to understand. If we use Ngoko style, we don't think it is appropriate. We are afraid if it (the Ngoko language) will hurt them (the elderly patients). That is the problem. We know we should respect the elderly, but we couldn't speak Kromo language well (R.1)."

"We should use 'appropriate' language, which is Kromo language since there are social norms to be considered. We are afraid of becoming impolite if we speak in Ngoko language (R.2)."

"We usually adjust the way we talk when we interacting with elderly patient. If they (elderly patients) speak in "Ngoko" style, that is really okay for us. But when they use Kromo language, we are really in trouble. We will be shamed if we can't adjust our self to use the similar style (R.5)."

\subsection{Category 3: Bridging the gap}

This category highlights strategies used by students to overcome communication barrier with elderly patients. Communication gap, primarily lack in "Kromo" language proficiency is perceived to cause ineffective communication between students and elderly patients. Thus, students use "bridge" to close the gap. They mobilized family and friends to "bridge" the communication.

"Most often, we asked family member (of the patient) for help. We explained what we want or what what we are going to do and then they (family members) will re-explain it to the patient. It was like that. They are just like a medium (R.1)."

"Sometimes, the family members have deeper understanding of what patient said compared to us.

Thus we use them as a medium to communicate with the patient (R.2)."

"My friend is from West Java. She was really in trouble when she has to see elderly patient who couldn't speak Bahasa. The patient couldn't understand what she said. She really needs a translator. She usually asks another student (who could speak Java) for companion (R.3)."

"Sometimes, we ask our friend (who could speak Java) to accompany us when we have to see patient. If they are not available, we will talk to the family members instead (R.4)."

Other techniques used by students to achieve successful communication with elderly were using simple sentences, using non-verbal language (gesture and facial expression) and reducing physical distant to patient.

"So, when we had to talk to elderly patient, we moved closer or showed our mimic (expression). It was like that (R.2)." 
"If we talked to adult patient, we like to keep distant at certain level. But when we talked to elderly patient, we come closer just to show that we care. Sometimes we touch their hands to express our caring when we care for female elderly patients (R.1)."

"If we want to explain something to patient, we have to make sure that it (the language) should be easy to understand. We shouldn't use difficult terms (R.5).",

\subsection{Category 4: Intimate connection}

This last category depicts positive view of students toward communication with elderly amid the challenges. One participant of this study found kind of "sense of intimacy" when interacting with elderly patients. This sense couldn't be found when interacting with client from other age groups.

For a matter of communication, I admit that caring for adult patient is far easier (compared to elderly patient). They have very good comprehension, their body function is still good and they could also understand many languages (not only Jawa language). However, at some point, I experience different feeling when I care for elderly patient. I couldn't really explain what it is. But I feel "close". It couldn't be found when you care for adult patient (R.6).

Hmm...I don't really know how to say my feeling. I just feel at ease, feel very comfortable (when communicate to elderly patient). I really have very good time with them (elderly patients). Most of the time, I experience all those feelings when I care for elderly patient (R.6).

\section{Discussion}

This study aimed to gain a better understanding of challenges faced by students nurse when communicating with elderly clients during clinical practice. The present study found that communication with elderly patients was apparently more challenging than communication with individual from other age groups. This finding supported the tendency as suggested by previous studies that inter-generational (different age group) communication is apparently more challenging than intra-generational (the same age group) communication. A quantitative study suggested that conversation with nonfamily elders was perceived as least satisfying, followed by family elder and sameage peers [9]. Age difference was found to be one of influential factors affecting communication between nurses and patients [10].

In this study barriers for an effective communication with elderly patients were perceived to be from either the patients or students nurses themselves. Patient related factors are including declining in hearing and cognitive function, while students' related factor is lack in "Kromo" language skill.

In communication with elderly patients, participants in this study found that declining the hearing function and thinking ability affects patients' speech perception and daily communication. Hearing impairment is one of the main problems in aging. The majority of hearing loss in older adult is sensory neural [11]. Few characteristics of age-related hearing loss are high frequency hearing loss, reduced hearing sensitivity and speech understanding in noisy environment. Hearing loss could have a great impact on elderly's daily activities, cognition, relationships, psychological health, and quality of life [12].

Effective communication also relies on an intact cognitive function. With normal 
aging, certain types of memory decline and cognitive processing slows. The length and complexity of sentences is also reduced [12].

Communication is heavily influenced by culture. Cultural groups have distinct linguistic and communication patterns. These patterns reflect core cultural values of a society [4]. Nurses are expected to have general knowledge of other cultures, especially those commonly encountered in their work area and its related skills to ensure the delivery of culturally congruent care.

Language commonality was seen as an important factor for effective communication [10]. However, in certain cultures where hierarchical language is applied, like in Javanese culture, effective communication is goes beyond "sharing the similar language". In Javanese culture, communicating with elderly using Kromo language is an expression of respect. Elderly in Javanese social structure is a group that earns an individual "hormat" (respect) from others. Hildred Geertz defines "hormat" as a Javanese term that means the recognition of superior rank by means of the appropriate forms of etiquette [13]. In Javanese culture, age is one of standard to define the rank. Nurses are typically younger than older adult patients. As a consequence, in this intergenerational-type communication it is crucial for them to show the respect by choosing the language level spoken.

There are numerous factors causing communication problem with older adult patients. Thus, it is important to provide mechanisms to help with communication issues such as adapting the environment, using assistive listening device and having communication partner adjust how they communicate with the individual [12]. Participants in this study have modified the way they communicate with elderly patient with hearing problem by positioning themselves close to the patients to reduce the distance and using non-verbal language such as gesture, facial expression and touch. Touch is one of the most effective forms of communication. It delivers many messages such as encouragement, affection, emotional support, and attention [4]. Meanwhile, when communicate with elderly patients with cognitive decline, it is necessary to communicate directly to topic with simple statement and provide adequate time to perceive, process, and respond to information [12].

Furthermore, in order to facilitating effective communication, companions during interaction may give some benefits. A previous study found that companions made a significant contribution to the communication between patient and health care personnel [14]. In this study, companions are family and friends who are able to speak Kromo to overcome language barrier.

Communication with elderly, from the participant's experience, may create the feeling of intimacy and comfort. This is an interesting finding though majority of students of this study didn't have the similar experience. To feel close and comfortable with our communication partner, these would be definitely helpful for an effective communication. Previous study had identified that lack of intimacy and official behavior were the most important nurse-related factors affecting communication [7]. Another study suggested that nurse' reluctant to communicate with the patients was one of important barriers, particularly from the viewpoint of patients [10]. It is expected that the presence of intimate or closeness feeling when interacting with elderly patient could give students positive experience which is important in building positive view and counter negative stereotype of elderly people. 


\section{Conclusion}

This study explored the experience of nursing students' communication with elderly patient. The present results indicated that communication barriers could be either from patient or nurse's students. Decline in sensory and cognitive functions of elderly patients and lack of local language proficiency by students nurse were perceived to be barriers for effective communication. Thus, to overcome these barriers, students applied certain techniques and used help from patient's family members or friends. Finally, amidst the challenges; however, students viewed that interaction with elderly patients was pleasing and comforting.

The findings of this study provided larger perspective of intergenerational communication in the context of nursing practice in multicultural society. With the growing number of elderly patients, nurse should become skilled at communication technique and basic local language spoken by older adult patient. Cultural awareness should be developed considering that Indonesia is a multicultural country. Developing constructive view of elderly people is also essential for successful communication.

\section{References}

[1] T. Dall, P. Gallo, R. Chakrabart, T. West, A. Semilla and M. Storm, Health Aff. 32, 2013-2020 (2013)

[2] J. Ansah, R. Eberlein, S. Love, M. Bautista, J. Thompson and Malhotra, Health Policy (New. York). 116, 105-13 (2014)

[3] L. Kourkouta and I. Papathanasiou,. Socio Medica. 26, 65-7

[4] P. Potter, A. Perry, P. Stockert and A. Hall Fundamental of Nursing (St. Louis, Missouri: Elsevier Mosby, 2013)

[5] C. Charlton, K. Dearin, J. Berry and M. Jhonson, J. Am. Assoc. Nurse Pract. 20, 382-8 (2008)

[6] J. Wang, P. Hsieh and C. Wang, Korean. Soc. Nurs. Sci. 7, 99-103 (2013)

[7] M. Agha and L. Hasani, J. Hormozgan Univ. 14, 312-8 (2011)

[8] K. Charmaz, Constructing Grounded Theory: A Practical Guide through Qualitative Analysis (London: SAGE Publication, 2006)

[9] H. Giles, K. Noels, A. William, H. Ota, T. Lim and S. Ng, J. Cross Cult. Gerontol. 18, (2003)

[10] Norouzinia R, Aghabarari M, Shiri M, Karimi M and Samami E 2016 Communication barriers perceived by nurses and patients Glob. J. Health Sci. 8 65-74

[11] S. Grossman and C. Porth, Porth's Pathophysiology: Concept of Altered Health States (Philadelphia: Lippincott Williams \& Wilkins, 2014)

[12] K. Mauk, Gerontological Nursing Competencies for Care (USA: Jones and Bartlett Publisher, 2010)

[13] D. Irawanto, P. Ramsey and J. Ryan, Challenge of leading in Javanese culture Asian Ethn. 12, 125-39 (2011)

[14] H. Ishikawa, D. Roter, Y. Yamazaki and T. Takayama, Soc. Sci. Med. 60, 230720 (2005) 